\title{
Twist gap and global symmetry in two dimensions
}

\author{
Nathan Benjamin $\odot,{ }^{1, *}$ Hirosi Ooguri $\odot,{ }^{2,3, \dagger}$ Shu-Heng Shao $\odot,{ }^{4, \$}$ and Yifan Wang ${ }^{5,6,8}$ \\ ${ }^{1}$ Princeton Center for Theoretical Science, Princeton University, Princeton, New Jersey 08544, USA \\ ${ }^{2}$ Walter Burke Institute for Theoretical Physics, California Institute of Technology, \\ Pasadena, California 91125, USA \\ ${ }^{3}$ Kavli Institute for the Physics and Mathematics of the Universe (WPI), University of Tokyo, \\ Kashiwa 277-8583, Japan \\ ${ }^{4}$ School of Natural Sciences, Institute for Advanced Study, Princeton, New Jersey 08540, USA \\ ${ }^{5}$ Center of Mathematical Sciences and Applications, Harvard University, \\ Cambridge, Massachusetts 02138, USA \\ ${ }^{6}$ Jefferson Physical Laboratory, Harvard University, Cambridge, Massachusetts 02138, USA
}

(Received 14 March 2020; accepted 13 May 2020; published 26 May 2020)

\begin{abstract}
We show that every compact, unitary two-dimensional conformal field theory with an Abelian conserved current has vanishing twist gap for charged primary fields with respect to the $\mathfrak{u}(1) \times$ Virasoro algebra. This means that either the chiral algebra is enhanced by a charged primary field with zero twist or there is an infinite family of charged primary fields that accumulate to zero twist.
\end{abstract}

DOI: 10.1103/PhysRevD.101.106026

\section{INTRODUCTION}

The study of two-dimensional (2D) conformal field theories (CFTs) has a long history in theoretical physics. Although much work has been done in understanding rational conformal field theories (RCFTs), ${ }^{1}$ there is very little general understanding of a generic, irrational 2D CFT. One feature that can diagnose irrationality of a CFT is the twist gap of the theory with respect to a certain chiral algebra. The twist, $2 t_{\mathcal{O}}$, of an operator $\mathcal{O}$ with total conformal dimension $\Delta$ and spin $j$ is defined as

$$
2 t_{\mathcal{O}}=\Delta-|j|=2 \min (h, \bar{h}),
$$

where $h$ and $\bar{h}$ are the left- and right-moving conformal dimensions of the operator. The twist gap of a theory is the minimum (or infimum) twist of all nonvacuum primary operators under the chiral algebra. For instance, if a CFT has a conserved current as a primary operator with respect to a certain chiral algebra, the twist gap of that CFT

*nathanb@princeton.edu

†oguri@caltech.edu

"shao@ias.edu

\$yifanw@g.harvard.edu

${ }^{1}$ Here we take the definition of RCFTs as 2D CFTs with a finite number of conformal blocks under a certain chiral algebra.

Published by the American Physical Society under the terms of the Creative Commons Attribution 4.0 International license. Further distribution of this work must maintain attribution to the author(s) and the published article's title, journal citation, and DOI. Funded by SCOAP ${ }^{3}$. vanishes under that chiral algebra. Given any chiral algebra, all rational CFTs must have vanishing twist gap under that chiral algebra for sufficiently large central charge. To be precise, if the vacuum Verma module of a chiral algebra grows asymptotically as a $c=c_{*}$ CFT, then all RCFTs with $c>c_{*}$ must have extra currents (and therefore vanishing twist gap) under that chiral algebra. ${ }^{2}$ Moreover, if a CFT has exactly marginal operators, and RCFTs are dense in the conformal manifold (for instance, in the case of the moduli space of the $c=1$ free boson which we discuss more below), then the twist gap under the chiral algebra shared by all theories in the moduli space must vanish everywhere.

In Sec. 2.2 of [2], a rigorous upper bound was placed on the twist gap of any $c>1$ compact, unitary CFT under the Virasoro algebra. ${ }^{3}$ It was shown that

$$
2 t_{\text {gap }}^{\text {Virasoro }} \leq \frac{c-1}{12} .
$$

In [3], it was argued that this bound can be improved to

\footnotetext{
${ }^{2}$ To be more precise, we define $c_{*}$ of a chiral algebra as follows. We say the number of vacuum descendants at dimension $\Delta$ of the chiral algebra goes as $e^{2 \pi \sqrt{\frac{c_{*} \Delta}{6}}}$ at large $\Delta$, up to subexponential corrections. For $c>c_{*}$, the Cardy formula [1] shows the number of primary operators under the chiral algebra goes as $e^{2 \pi \sqrt{\frac{\left(c-c_{*}\right) \Delta}{3}}}$. Thus, if $c>c_{*}$, there must be infinitely many primary operators under this chiral algebra, which means that the RCFT must have additional currents.

${ }^{3}$ In [2], the argument was credited to Tom Hartman.
} 


$$
2 t_{\mathrm{gap}}^{\text {Virasoro }} \leq \frac{c-1}{16}
$$

from positivity of the spectrum of the theory. We emphasize that both of these bounds are nonzero, and indeed it is expected that a generic interacting CFT has a finite, nonzero twist gap under the Virasoro algebra (although we pause to note that we do not know of any explicit example of such a theory). The latter improved twist gap bound was recently revisited in [4] using a Rademacher expansion of the torus partition function.

In this paper, we will show that in any compact CFT with an Abelian conserved current $(J, \bar{J})$ whose holomorphic Fourier modes $J_{n}$ generate the $\mathfrak{t}(1)$ chiral algebra (similarly for the antiholomorphic part), ${ }^{4}$ the twist gap under the $\mathfrak{t}(1) \times$ Virasoro algebra vanishes,

$$
2 t_{\text {gap }}^{\mathfrak{t}(1) \times \text { Virasoro }}=0 .
$$

Thus, the situation for the $\mathfrak{t}(1)$ current algebra is in stark contrast to the Virasoro algebra.

Note that the holomorphic current $J$ (or the antiholomorphic component $\bar{J}$ ) generates either a compact $U(1)$ or $\mathbb{R}$ symmetry, and we do not make assumptions on this in the paper. In other words, we show that there is always a charged primary operator under the $\mathfrak{t}(1)$ current algebra that is either a conserved (higher-spin) current, or an infinite family of charged primary operators that accumulate to zero twist.

The prototypical example is the $c=1$ compact boson at radius $R$. When $R^{2}$ is rational, there is a holomorphic current extending the $\mathfrak{t}(1) \times$ Virasoro algebra, and the twist gap with respect to the $\mathfrak{u}(1) \times$ Virasoro algebra vanishes. On the other hand, when $R^{2}$ is irrational, there is a tower of (nonholomorphic) primary operators that asymptote to vanishing twist.

As an application, we also show that any $c>3$ CFT with $\mathcal{N}=2$ superconformal symmetry has a vanishing twist gap under the (unextended) $\mathcal{N}=2$ super-Virasoro algebra.

\section{II. $U(1)$ GLOBAL SYMMETRY}

In this section, we discuss properties of 2D CFTs with compact $U(1)$ global symmetry (the compactness assumption will be relaxed in the next section).

\section{A. Currents and charges}

Consider a 2D compact, unitary, bosonic CFT with a $U(1)$ global symmetry. Being a bosonic CFT, all local

\footnotetext{
${ }^{4}$ We use $\mathfrak{t}(1)$ for a rank-one Abelian Lie algebra, and we reserve $U(1)$ for a compact Abelian Lie group. When the holomorphic current $J$ has a preferred normalization (e.g., when its charges are quantized), we will denote the corresponding chiral algebra by $\mathfrak{t}(1)_{k}$, where $k$ is the level of the current algebra, and similarly for the antiholomorphic current $\bar{J}$.
}

operators have integral Lorentz spin, $h-\bar{h} \in \mathbb{Z}$. We will later generalize our arguments for fermionic CFTs. It will be important that the global structure of the symmetry is $U(1)$ not $\mathbb{R}$.

Consider a $U(1)$ global symmetry generated by the current $J_{\mu}(z, \bar{z})$ that acts faithfully on the Hilbert space. Let the holomorphic and antiholomorphic components of the current be $J(z) \equiv J_{z}$ and $\bar{J}(\bar{z}) \equiv J_{\bar{z}}$, respectively. In any compact unitary two-dimensional CFT, current conservation and unitarity imply $\partial \bar{J}=0$ and $\bar{\partial} J=0$. However, the holomorphic current $J(z)$ alone may not necessarily generate a compact $U(1)$ group, but $\mathbb{R}$ rather, and similarly for the antiholomorphic current $\bar{J}$.

The conserved charge $U_{\eta}$ with $\eta \in[0,1)$ for this $U(1)$ global symmetry is supported on a curve $L$ in the Euclidean spacetime,

$$
U_{\eta}=: \exp \left[\eta\left(\oint_{L} d z J(z)-\oint_{L} d \bar{z} \bar{J}(\bar{z})\right)\right]:,
$$

where : stands for normal ordering. Current conservation $\partial \bar{J}=\bar{\partial} J=0$ implies that $U_{\eta}$ is invariant under small deformation of the curve $L$. Hence, $U_{\eta}$ is a topological line operator. See, for example, [5-7] for discussions on topological lines in two dimensions. The $U(1)$ condition implies that $\eta$ is circle valued i.e., $\eta \sim \eta+1$. In particular, $U_{\eta=1}=I$ is the identity operator on the Hilbert space. The faithfulness condition implies that $U_{\eta}$ is not an identity operator unless $\eta$ is an integer.

The operator product expansions (OPEs) of $J$ and $\bar{J}$ are

$$
J(z) J(0) \sim \frac{k}{z^{2}}, \quad \bar{J}(\bar{z}) \bar{J}(0) \sim \frac{\bar{k}}{\bar{z}^{2}} .
$$

Having specified a faithful $U(1)$ global symmetry, the levels $k$ and $\bar{k}$ are physically meaningful and cannot be scaled away. ${ }^{5}$

\section{B. Spectral flow and the twisted Hilbert Space}

When $U_{\eta}$ is supported on the whole space at a fixed time, it is an operator acting on the Hilbert space $\mathcal{H}$. On the other hand, when $U_{\eta}$ is supported at a fixed position in space but extends in time, it is a defect. The insertion of this defect gives rise to a twisted boundary condition for the quantization. We will denote the Hilbert space on a circle $S^{1}$ with an insertion of a defect $U_{\eta}$ as $\mathcal{H}_{\eta}$. Via the operator-state correspondence, a state in $\mathcal{H}_{\eta}$ is mapped to a nonlocal, pointlike operator living at the end of the topological line

\footnotetext{
${ }^{5}$ For example, if we had rescaled both $J \rightarrow 2 J$ and $\bar{J} \rightarrow 2 \bar{J}$, then the new topological line operator $U_{\eta}$ with $\eta=1 / 2$ would act trivially on the Hilbert space $\mathcal{H}$, violating the faithfulness condition.
} 


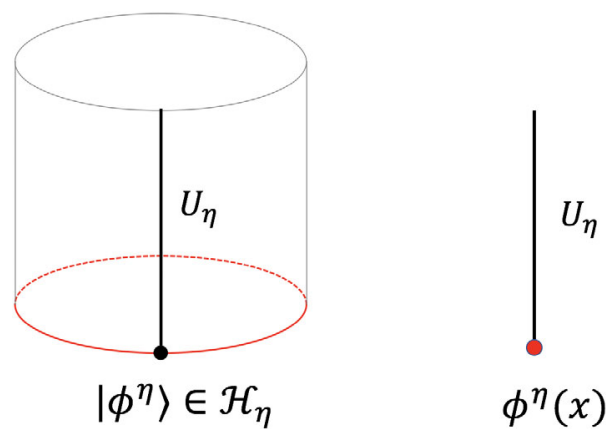

FIG. 1. Using the operator-state correspondence, a state $\left|\phi^{\eta}\right\rangle$ in the twisted Hilbert space $\mathcal{H}_{\eta}$ is mapped to a nonlocal, pointlike operator attached to a topological line defect $U_{\eta}$.

$U_{\eta}$ on the plane (see Fig. 1). In particular, $\mathcal{H} \equiv \mathcal{H}_{\eta=0}$ is the original Hilbert space of local operators.

What is the relation between the twisted Hilbert space $\mathcal{H}_{\eta}$ and the Hilbert space of local operators $\mathcal{H}$ ? This is explained by the spectral flow, which is an isomorphism between $\mathcal{H}_{\eta}$ and $\mathcal{H}$ [7]. More precisely, spectral flow maps a state $|\phi\rangle \in \mathcal{H}$ with quantum numbers $\left(L_{0}, \bar{L}_{0}, J_{0}, \bar{J}_{0}\right)$ to a state in $\left|\phi^{\eta}\right\rangle \in \mathcal{H}_{\eta}$ with quantum numbers $\left(L_{0}^{\eta}, \bar{L}_{0}^{\eta}, J_{0}^{\eta}, \bar{J}_{0}^{\eta}\right)[8]$,

$$
\begin{array}{ll}
L_{0}^{\eta}=L_{0}-\eta J_{0}+\frac{k \eta^{2}}{2}, & J_{0}^{\eta}=J_{0}-\eta k, \\
\bar{L}_{0}^{\eta}=\bar{L}_{0}+\eta \bar{J}_{0}+\frac{\bar{k} \eta^{2}}{2}, & \bar{J}_{0}^{\eta}=\bar{J}_{0}+\eta \bar{k} .
\end{array}
$$

Spectral flow is related to the modular property of the partition function, as we demonstrate in the following. Consider the torus partition function with general chemical potentials for the symmetry generators $J_{0}$ and $\bar{J}_{0}$,

$$
\begin{aligned}
Z(\tau, \bar{\tau}, \eta, \bar{\eta})= & \operatorname{Tr}_{\mathcal{H}} \exp \left[2 \pi i \tau\left(L_{0}-\frac{c}{24}\right)-2 \pi i \bar{\tau}\left(\bar{L}_{0}-\frac{c}{24}\right)\right. \\
& \left.+2 \pi i \eta J_{0}-2 \pi i \bar{\eta} \bar{J}_{0}\right] .
\end{aligned}
$$

The torus partition function satisfies the modular property [9]

$$
\begin{aligned}
& Z(-1 / \tau,-1 / \bar{\tau}, \eta / \tau, \bar{\eta} / \bar{\tau}) \\
& \quad=\exp \left(i k \pi \frac{\eta^{2}}{\tau}-i \bar{k} \pi \frac{\bar{\eta}^{2}}{\bar{\tau}}\right) Z(\tau, \bar{\tau}, \eta, \bar{\eta}) .
\end{aligned}
$$

Let us understand this modular property in the special case when $\bar{\eta}=-\eta$. In this case, the partition function $Z(\tau, \bar{\tau}, \eta)$ on the right-hand side is the trace over the Hilbert space $\mathcal{H}$ with $U_{\eta}$ inserted at a fixed time,

$$
\begin{aligned}
Z(\tau, \bar{\tau}, \eta, \bar{\eta}=-\eta)= & \operatorname{Tr}_{\mathcal{H}}\left\{U _ { \eta } \operatorname { e x p } \left[2 \pi i \tau\left(L_{0}^{\eta}-\frac{c}{24}\right)\right.\right. \\
& \left.\left.-2 \pi i \bar{\tau}\left(\bar{L}_{0}^{\eta}-\frac{c}{24}\right)\right]\right\} .
\end{aligned}
$$

It also admits an $S$-dual interpretation as the partition function over the twisted Hilbert space $\mathcal{H}_{\eta}$ on a torus with modulus $-1 / \tau$,

$$
\begin{aligned}
& Z(\tau, \bar{\tau}, \eta, \bar{\eta}=-\eta) \\
& \quad=\operatorname{Tr}_{\mathcal{H}_{\eta}} \exp \left[-\frac{2 \pi i}{\tau}\left(L_{0}^{\eta}-\frac{c}{24}\right)+\frac{2 \pi i}{\bar{\tau}}\left(\bar{L}_{0}^{\eta}-\frac{c}{24}\right)\right] .
\end{aligned}
$$

The left-hand side of (2.5), on the other hand, can be written as a sum over the original Hilbert space,

$$
\begin{aligned}
Z(-1 / \tau,-1 / \bar{\tau}, \eta / \tau,-\eta / \bar{\tau}) \\
=\operatorname{Tr}_{\mathcal{H}} \exp \left[-\frac{2 \pi i}{\tau}\left(L_{0}-\frac{c}{24}\right)+\frac{2 \pi i}{\bar{\tau}}\left(\bar{L}_{0}-\frac{c}{24}\right)\right. \\
\left.\quad+2 \pi i\left(\frac{\eta}{\tau} J_{0}+\frac{\eta}{\bar{\tau}} \bar{J}_{0}\right)\right] .
\end{aligned}
$$

Using the expressions (2.7) and (2.8), we see that (2.5) is reproduced by the spectral flow map (2.3).

So far, we have not used the fact that the underlying symmetry is globally $U(1)$ instead of $\mathbb{R}$. If the global structure is $U(1)$, then the charge $U_{\eta}$ is trivial when $\eta \in \mathbb{Z}$. In particular, the twisted Hilbert space $\mathcal{H}_{\eta=1}$ reduces to the Hilbert space $\mathcal{H}$ of local operators. In other words, spectral flow with integer units $\eta \in \mathbb{Z}$ maps a local operator to another. See Fig. 2. This property would not have held if the global structure of the symmetry is given by $\mathbb{R}$ instead of $U(1)$.

Let us study the consequences of the $U(1)$ symmetry. From (2.3), the operator $\phi^{\eta}$ obtained from the spectral flow has Lorentz spin,

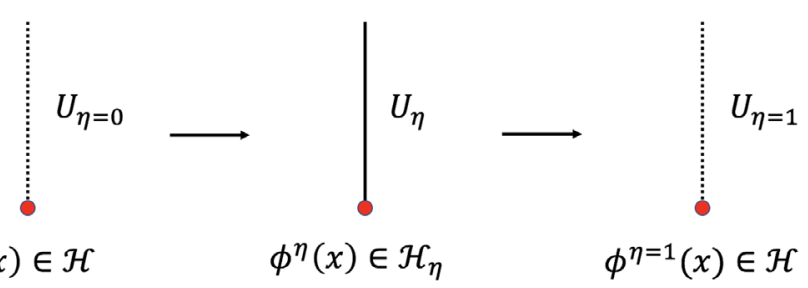

FIG. 2. Starting with a local operator $\phi(x) \in \mathcal{H}$, we can adiabatically turn on a topological line $U_{\eta}$ from $\eta=0$ (the trivial line, shown in dashed line) to a small but finite value of $\eta$. This implements the spectral flow map from a local operator $\phi(x)$ to a nonlocal one $\phi^{\eta}(x)$ living at the end of the line. When $\eta$ increases to 1 (corresponding to the $2 \pi$ rotation of $U(1)$ ), the topological line becomes trivial, and we end up with another local operator $\phi^{\eta=1}(x) \in \mathcal{H}$. The whole process implements a spectral flow by one unit that maps a local operator $\phi(x)$ to another $\phi^{\eta=1}(x)$. 


$$
L_{0}^{\eta}-\bar{L}_{0}^{\eta}=\left(L_{0}-\bar{L}_{0}\right)-\eta\left(J_{0}+\bar{J}_{0}\right)+\frac{\eta^{2}}{2}(k-\bar{k}) .
$$

Now, let us assume $\eta \in \mathbb{Z}$, so the operator $\phi^{\eta}$ is a local operator. In a bosonic CFT, since the original local operator $\phi$ has integral Lorentz spin and $U(1)$ charge i.e., $L_{0}-\bar{L}_{0} \in \mathbb{Z}$ and $J_{0}+\bar{J}_{0} \in \mathbb{Z}$, the quantization of the Lorentz spin for $\phi^{\eta}$ implies that the levels have to obey

$$
\text { (bosonic): } k-\bar{k} \in 2 \mathbb{Z},
$$

which is the 't Hooft anomaly of the $U(1)$ global symmetry. If the CFT is fermionic, the Lorentz spin can be half integer and the quantization of the levels is modified to

$$
\text { (fermionic) }: k-\bar{k} \in \mathbb{Z} \text {. }
$$

\section{Example: Compact boson}

Let us illustrate the above general discussions in the example of $c=1$ compact boson at radius $R$. Let $X(z, \bar{z})=$ $X_{L}(z)+X_{R}(\bar{z})$ be the compact boson field with the identification $X(z, \bar{z}) \sim X(z, \bar{z})+2 \pi R$. We normalize the OPE to be

$$
X(z, \bar{z}) X(0,0) \sim-\frac{1}{2} \log |z|^{2} .
$$

Hence, $\partial X(z) \partial X(0) \sim-\frac{1}{2 z^{2}}$ and $\bar{\partial} X(\bar{z}) \bar{\partial} X(0) \sim-\frac{1}{2 \bar{z}^{2}}$. The $\mathfrak{t}(1)_{k} \times$ Virasoro primaries are the exponential operators,

$\mathcal{O}_{n, w}(z, \bar{z})=\exp \left[i\left(\frac{n}{R}+w R\right) X_{L}(z)+i\left(\frac{n}{R}-w R\right) X_{R}(\bar{z})\right]$,

with integer momentum $n$ and winding numbers $w$. The conformal weights are

$$
h=\frac{1}{4}\left(\frac{n}{R}+w R\right)^{2}, \quad \bar{h}=\frac{1}{4}\left(\frac{n}{R}-w R\right)^{2} .
$$

We have the OPE,

$$
\begin{aligned}
i \partial X(z) \mathcal{O}_{n, w}(0) & \sim \frac{\left(\frac{n}{R}+w R\right)}{2 z} \mathcal{O}_{n, w}(0), \\
i \bar{\partial} X(\bar{z}) \mathcal{O}_{n, w}(0) & \sim \frac{\left(\frac{n}{R}-w R\right)}{2 \bar{z}} \mathcal{O}_{n, w}(0) .
\end{aligned}
$$

For generic $R$, the charges of $i \partial X(z)$ and $i \bar{\partial} X(\bar{z})$ are irrational. This implies that they generate two $\mathbb{R}$ groups instead of $U(1)$.

\footnotetext{
${ }^{6}$ Our convention for the compact boson radius is such that $R=1$ corresponds to the self-dual point with enhanced $\mathfrak{g} \mathfrak{t}(2)$ current algebra.
}

At any radius $R$, there are two $U(1)$ global symmetries, the momentum $U(1)_{n}$ and the winding $U(1)_{w}$, that are not holomorphic nor antiholomorphic. The currents of the momentum and winding $U(1)$ 's are combinations of $\partial X(z)$ and $\bar{\partial} X(\bar{z})$,

$U(1)_{n}: J(z)=i R \partial X(z), \quad \bar{J}(\bar{z})=i R \bar{\partial} X(\bar{z})$,

$U(1)_{w}: J(z)=\frac{i}{R} \partial X(z), \quad \bar{J}(\bar{z})=-\frac{i}{R} \bar{\partial} X(\bar{z})$,

under which $\mathcal{O}_{n, w}$ has integer charges $n$ and $w$, respectively. The levels $k$ and $\bar{k}$ of the two $U(1)$ 's are

$$
\begin{gathered}
U(1)_{n}: k=\bar{k}=\frac{R^{2}}{2}, \\
U(1)_{w}: k=\bar{k}=\frac{1}{2 R^{2}} .
\end{gathered}
$$

In particular, they both obey $k=\bar{k}$, which means that they are nonanomalous (but there is a mixed anomaly between them). Note that $k$ and $\bar{k}$ are not separated quantized in general. When $R^{2}$ is rational, there is an integral linear combination of $U(1)_{n}$ and $U(1)_{w}$ that is holomorphic and another integral combination that is antiholomorphic. By contrast, at an irrational radius, there is no holomorphic nor antiholomorphic $U(1)$ global symmetry, but only $\mathbb{R}$.

The spectral flow (2.3) for $U(1)_{n}$ by one unit $\eta=1$ maps the exponential operator $\mathcal{O}_{n, w}$ to $\mathcal{O}_{n, w-1}$, while the spectral flow for $U(1)_{w}$ maps $\mathcal{O}_{n, w}$ to $\mathcal{O}_{n-1, w}$.

\section{VANISHING TWIST GAP}

\section{A. Compactness of global symmetry}

We will show, under some assumption which we will spell out later, that a current $(J, \bar{J})$ acting on a CFT with a discrete spectrum generates a compact $U(1) \times U(1)$ symmetry as opposed to $U(1) \times \mathbb{R}$ or $\mathbb{R} \times \mathbb{R}^{7}$. For definiteness, let us first assume that there are no other conserved

\footnotetext{
${ }^{7}$ It is also possible that the rank of the symmetry is one. This happens, for example, when $\bar{k}=0$ and $\bar{J}$ vanishes identically. In this case, a straightforward generalization of the following argument shows that the symmetry group is $U(1)$ rather than $\mathbb{R}$, and our argument for the vanishing twist gap in Sec. III B will follow. There is also a logical possibility that $k$ and $\bar{k}$ are both nonzero, but the left- and right-moving charges are correlated (i.e., $J_{0}=C \bar{J}_{0}$ for some nonzero constant $C$ ) in such a way that the rank of the global symmetry is still one (e.g., the $c=1$ free boson with only momentum modes and no winding modes). However, this does not happen for a compact CFT. If the rank were one, a straightforward generalization of the following argument shows that the left-moving charges are all rational numbers with a common denominator, which means the leftmoving $U(1)$ symmetry is compact. Then the spectral flow operators (3.1) of this $U(1)$ (which also follow from modular covariance) would violate $J_{0}=C \bar{J}_{0}$ and hence a contradiction.
} 
currents, though our argument can be easily generalized to cases with additional currents.

Let us first look at the spectrum of the left-moving charge $J_{0}$. Assuming faithfulness, there must be at least one operator with a nonzero charge. Let us normalize $J(z)$ so that the lightest charged state carries $J_{0}=1$. We claim that all other charges can be written as either as $J_{0}=q$ with $q \in \mathbb{Q}$ or $J_{0}=q_{1}+x q_{2}$, where $q_{1}, q_{2} \in \mathbb{Q}$ and $x$ is some irrational number. To show this, suppose to the contrary that there were a state with $J_{0}=y$ for some irrational $y$ that cannot be written as $y=q_{1}+x q_{2}$. We would then need at least three rational numbers $q_{1}, q_{2}$, and $q_{3}$ to parametrize $J_{0}$ eigenvalues as $J_{0}=q_{1}+x q_{2}+y q_{3}$. The operator product expansion would then preserve $q_{1}, q_{2}$, and $q_{3}$ separately. Thus, we would conclude that the rank of global symmetry preserving the spectrum and the operator product expansion is at least three. By the Noether theorem, there must then be at least three conserved currents, contradicting our assumption that there are no other conserved currents.

We can repeat the argument for the right-mover and conclude that the $\bar{J}$ spectrum must be either $\bar{J}_{0}=p$ with $p \in \mathbb{Q}$ or $\bar{J}_{0}=p_{1}+x p_{2}$ with $p_{1}, p_{2} \in \mathbb{Q}$. We must have the same irrational number $x$ for both $J$ and $\bar{J}$; otherwise, there must be at least three conserved currents. Moreover, the spectra of $J_{0}$ and $\bar{J}_{0}$ must be correlated; we must be able to take their two linearly independent combinations such that their eigenvalues are both in $\mathbb{Q}$. If it is not possible to take such linear combinations, the rank of the global symmetry must be greater than two, contradicting our assumption.

We have been able to show that if a current $(J, \bar{J})$ acts faithfully on a CFT and if there are no other conserved currents, then there must be two linearly independent combinations of $J_{0}$ and $\bar{J}_{0}$ such that their eigenvalues are all rational. In order for these two combinations to generate a compact $U(1) \times U(1)$ symmetry, these rational charges must have their denominators bounded. If the CFT has a discrete spectrum, then for any $\Delta>0$, there are a finite number of states with conformal dimensions below $\Delta$ and therefore the denominators are bounded. The question is what happens when we take the limit $\Delta \rightarrow \infty$.

In Sec. 6 of [10], Daniel Harlow and one of the authors showed that if a CFT spectrum is finitely generated, then any noncompact global symmetry of that CFT is a subgroup of a larger compact global symmetry. "Finitely generated" means that there is a finite subset of the primary fields whose operator product expansion generates all other primary fields (see [10] for a precise formulation). In our case, the proof is straightforward. Since rational charges of a finite subset of primary fields have bounded

\footnotetext{
${ }^{8}$ Here, we are discussing compactness applicable to general CFT not necessarily with a weakly coupled gravity dual. For compactness of gauge symmetry in gravitational theory, see also the earlier papers $[11,12]$.
}

denominators, so are all other charges generated by the operator product expansion. This shows that the global symmetry generated by $(J, \bar{J})$ is $U(1) \times U(1)$ or is $U(1)$ when either $J=0$ or $\bar{J}=0$.

We hope that the assumption that the CFT spectrum is finitely generated can be relaxed, at least in two dimensions.

\section{B. Twist gap and the $U(1)$ global symmetry}

Consider a 2D compact, unitary CFT with a $U(1)$ global symmetry generated by the current $(J, \bar{J})$. Unitarity and current conservation imply that both components of the current are conserved, so they separately generate two Abelian symmetries. In the previous section, we have argued that the full global symmetry associated to $(J, \bar{J})$ is either $U(1) \times U(1)$ or $U(1)$. Note that, in the rank two case, each $U(1)$ factor is not necessarily holomorphic or antiholomorphic. As reviewed in Sect. II C, this is the case for the $c=1$ free boson at a generic radius, where the leftand right-moving charges with respect to $J$ and $\bar{J}$ are irrational, while the momentum and winding charges are quantized.

As a warmup, let us show that the twist gap is zero for the special case when the symmetry group is $U(1)$ and is generated by a holomorphic current or an antiholomorphic current. Let us assume the $U(1)$ current is holomorphic, while the antiholomorphic case works identically. To avoid confusion, we denote the Abelian current algebra generated by the modes $J_{n}$ of a holomorphic current $J$ with level $k$ by $\mathfrak{t}(1)_{k}$. If the holomorphic global symmetry is globally $U(1)$, then $\bar{k}=0$ and the level has to be quantized as $k \in 2 \mathbb{Z}$ from (2.10). In this case, the spectral flow by \pm 1 unit (2.3) maps the identity operator to a pair of local operators with quantum number

$$
\begin{array}{llrl}
L_{0} & =\frac{k}{2}, & & J_{0}=\mp k, \\
\bar{L}_{0}=0, & \bar{J}_{0}=0 .
\end{array}
$$

Therefore, the current algebra is enhanced by these higher spin currents. These (higher-spin) spectral flow currents are necessarily $\mathfrak{u}(1)_{k}$ primaries. ${ }^{9}$ Consequently, any compact CFT with holomorphic $U(1)$ symmetry has a tower (and the complex conjugate) of $\mathfrak{t}(1)_{k}$ primaries of zero twist from the spectral flow currents.

It remains to show that the twist gap is zero when the holomorphic current generates an $\mathbb{R}$ symmetry instead of $U(1)$.

\footnotetext{
${ }^{9} \mathrm{We}$ prove this statement below. Suppose the spectral flow current associated to $\eta=1$ is an $\mathfrak{t}(1)_{k}$ descendant of a primary $\mathcal{O}$ with $J_{0}=-k$ and $L_{0}<k / 2$. Then we can apply another spectral flow with $\eta=-1$ on $\mathcal{O}$ to obtain a local operator with $J_{0}=0$ and $L_{0}<0$, which would violate unitarity.
} 
From Sec. III A, we conclude that the global form of the symmetry is $U(1) \times U(1)$. By assumption, one of the $U(1)$ symmetries is generated by the (nonholomorphic) current $(J, \bar{J})$. Let us assume the other $U(1)$ is generated by the current $(\alpha J, \beta \bar{J})$ for some real numbers $\alpha, \beta$ (with $\alpha \neq \beta$ ). Importantly, we assume $\alpha, \beta$ are irrational numbers. Otherwise an integer linear combination of the two compact $U(1)$ 's would generate a holomorphic $U(1)$ symmetry and our previous argument would be sufficient to show vanishing twist gap. The levels of the second $U(1)$ are $\left(\alpha^{2} k, \beta^{2} \bar{k}\right)$. For each $U(1)$ factor, we can perform independent spectral flows as explained in Sec. II B. ${ }^{10}$

With respect to the first $U(1)$ symmetry, spectral flow by $\eta \in \mathbb{Z}$ units takes a state in the Hilbert space $\mathcal{H}$ to another with the following changes in quantum numbers given by (2.3). Meanwhile, spectral flow with respect to the second $U(1)$ by $\eta^{\prime} \in \mathbb{Z}$ units induces

$$
\begin{aligned}
L_{0} & \rightarrow L_{0}-\eta^{\prime} \alpha J_{0}+\frac{\eta^{\prime 2} \alpha^{2} k}{2} \\
\alpha J_{0} & \rightarrow \alpha J_{0}-\eta^{\prime} \alpha^{2} k \\
\bar{L}_{0} & \rightarrow \bar{L}_{0}+\eta^{\prime} \beta \bar{J}_{0}+\frac{\eta^{\prime 2} \beta^{2} \bar{k}}{2} \\
\beta \bar{J}_{0} & \rightarrow \beta \bar{J}_{0}+\eta^{\prime} \beta^{2} \bar{k},
\end{aligned}
$$

which follows from (2.3) with the replacement $J_{0} \rightarrow \alpha J_{0}$, $k \rightarrow \alpha^{2} k, \bar{J}_{0} \rightarrow \beta \bar{J}_{0}, \bar{k} \rightarrow \beta^{2} \bar{k}$.

Let us now take the vacuum state and spectral flow first by $\eta \in \mathbb{Z}$ units with respect to the first $U(1)$ and $\eta^{\prime} \in \mathbb{Z}$ units with respect to the second $U(1)$ (or vice versa). The resulting state will have quantum numbers,

$$
\begin{aligned}
L_{0}^{\eta, \eta^{\prime}} & =\frac{k}{2}\left(\eta+\eta^{\prime} \alpha\right)^{2} \\
\bar{L}_{0}^{\eta, \eta^{\prime}} & =\frac{\bar{k}}{2}\left(\eta+\eta^{\prime} \beta\right)^{2} \\
J_{0}^{\eta, \eta^{\prime}} & =-k\left(\eta+\eta^{\prime} \alpha\right) \\
\bar{J}_{0}^{\eta, \eta^{\prime}} & =\bar{k}\left(\eta+\eta^{\prime} \beta\right) .
\end{aligned}
$$

By choosing the integers $\eta, \eta^{\prime}$, we can have $\eta+\eta^{\prime} \alpha$ arbitrarily close to zero. ${ }^{11}$ Hence, we have shown that the twist gap of this theory must indeed vanish.

\footnotetext{
${ }^{10}$ For the $c=1$ free boson at radius $R$, if we take the momentum $U(1)_{n}$ to be the first nonholomorphic $U(1)$, then $k=\bar{k}=\frac{R^{2}}{2}, \alpha=\frac{1}{R^{2}}, \beta=-\frac{1}{R^{2}}$ (see Sec. II C).

${ }^{11}$ Here we have used Dirichlet's approximation theorem, which states that for any irrational number $\alpha$, the inequality $\left|\alpha+\eta / \eta^{\prime}\right|<\frac{1}{\left(\eta^{\prime}\right)^{2}}$ is satisfied for infinitely many integers $\eta, \eta^{\prime}$. We thank Petr Kravchuk and Juan Maldacena for discussions on this point.
}

Note that (3.2) implies the following conditions on the current levels from spin quantization (invariance of the theory under modular $T$ transformations):

$$
\begin{gathered}
\alpha k-\beta \bar{k} \in \mathbb{Z} \\
\alpha^{2} k-\beta^{2} \bar{k} \in 2 \mathbb{Z} .
\end{gathered}
$$

These theories all come with an exactly marginal operator, $J \bar{J}$. The quantization conditions (2.10) and (3.4) imply that under deformation by this operator, the parameters $\alpha, \beta, k, \bar{k}$ must change in a controlled way. In particular, if

$\alpha \rightarrow \alpha+\delta \alpha, \quad \beta \rightarrow \beta+\delta \beta, \quad k \rightarrow k+\delta k, \quad \bar{k} \rightarrow \bar{k}+\delta \bar{k}$

under a small deformation, then

$\delta k=\delta \bar{k}, \quad \delta \alpha=\frac{\beta-\alpha}{2 k} \delta k, \quad \delta \beta=\frac{\alpha-\beta}{2 \bar{k}} \delta k$,

where the first condition comes from (2.10). It can be checked that the free boson under a small deformation in $R$ obeys (3.6).

We pause here to note that we have two rather qualitatively different arguments to show the twist gap vanishes for all 2D CFTs with an Abelian current. The first is the holomorphic current $J$ is associated with a compact global symmetry $U(1)$. In this case, the spectral flow operation on the vacuum state maps to a new charged state with twist 0 that is not a vacuum descendent. The second is if the global $U(1) \times U(1)$ is generated by currents $(J, \bar{J})$ and $(\alpha J, \beta \bar{J})$ for irrational $\alpha, \beta$. In this case, we cannot show there must be a nontrivial primary of zero twist in the CFT, but we can show there is an infinite tower of states that accumulate to zero twist.

The latter situation that we described in the above paragraph is the generic situation for 2D CFTs with an Abelian current. If the global $U(1) \times U(1)$ symmetry is generated by currents $(J, \bar{J})$ and $(\alpha J, \beta \bar{J})$ for at least one of $\alpha, \beta$ rational, then we can simply deform by the $J \bar{J}$ operator which would change $\alpha, \beta$ as in (3.6) and generically make them both irrational. (E.g., $\alpha, \beta$ are generically irrational in the $c=1$ compact boson.) This means that a generic 2D CFT with an Abelian current has an accumulation point at zero twist. Since these operators have arbitrarily small but nonzero twist, they cannot be the vacuum descendants of any chiral algebra.

We end this section by remarking that compactness of the $U(1)$ symmetry is essential in our argument for a vanishing twist gap. The crossing equation (2.5) alone is not enough to prove a vanishing twist gap. As a counterexample, we can take the $\mathfrak{t}(1)_{k} \times$ Virasoro vacuum character and perform a regularized sum over modular images in the same fashion as [13,14]. This will give a finite, 
modular-invariant spectrum with a unique $\operatorname{PSL}(2, \mathbb{C})$ invariant vacuum, and a continuous energy spectrum at each spin, with continuous charges. From the modular kernels of the $\mathfrak{t}(1)_{k} \times$ Virasoro characters (see Appendix C of [9]), the spectrum will only have support when the twist is at least $\frac{c-2}{12} \cdot{ }^{12}$ This means the lightest charged states have a finite twist gap. Since the charge is continuous, this theory (if it exists) does not have compact $U(1)$ symmetries and is not finitely generated, and therefore does not contradict our result.

\section{Applications to $\mathcal{N}=2$ theories}

We now apply the results of Secs. III A and III B to the case of $\mathcal{N}=(2,2)$ superconformal field theories with $c>3$. Recall that the unextended $\mathcal{N}=2$ super-Virasoro algebra contains as generators, the supercurrents $G^{ \pm}(z)$, R-current $J_{R}(z)$ in addition to the stress tensor $T(z)$. We would like to show that any compact $c>3 \mathcal{N}=(2,2)$ superconformal field theory (SCFT) has a vanishing twist gap with respect to the unextended $\mathcal{N}=2$ super-Virasoro algebra. When $c<3$, there are $\mathcal{N}=2$ minimal models whose twist gaps are nonzero.

There are two cases to consider: the holomorphic R-symmetry is globally $U(1)$ or $\mathbb{R}$. We start with the case where the holomorphic R-symmetry is $U(1)$. Let the holomorphic level of the current $J_{R}(z)$ be $k$, normalized such that the $U(1)$ R-symmetry acts faithfully with all the states having integer charges. In particular, the level $k$ is the 't Hooft anomaly (2.11) of the $U(1)$ R-symmetry and has to be an integer, $k \in \mathbb{Z}$. This is different from the usual convention where the supercurrents have unit charges,

$$
q_{\text {usual }}\left(G^{ \pm}\right)= \pm 1
$$

while the charged operators might have fractional charges. Instead, we normalize the charges so that the supercurrents have

$$
q\left(G^{ \pm}\right)= \pm \sqrt{\frac{3 k}{c}} \in \mathbb{Z}
$$

Accordingly, the BPS condition in the NS sector is now

\footnotetext{
${ }^{12}$ Here we assumed $c>2$. In general, the vacuum $\mathfrak{t}(1)_{k} \times$ Virasoro character is the product the of $\mathfrak{t}(1)_{k}$ vacuum character $\chi_{0}^{\mathfrak{u}(1)_{k}}=\frac{q^{\frac{1}{24}}}{\eta(q)}$, and the Virasoro vacuum character $\chi_{0}^{\mathrm{Vir}}$ at central charge $c-1[15,16]$. For $c>2$, under S-transformation, the modular kernel has support for $h \geq \frac{c-2}{24}$ as shown in [9]. However, for $c \leq 2$, the modular S-transformation also has support at the

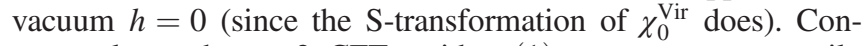
sequently, such $c<2$ CFTs with $\mathfrak{t}(1)$ symmetry necessarily have twist zero states (higher-spin currents).
}

$$
h=\sqrt{\frac{c}{12 k}} q
$$

rather than the more familiar $h=\frac{1}{2} q_{\text {usual }}$.

When the holomorphic R-symmetry is globally $U(1)$, we can apply an integer unit of spectral flow on the identity operator to obtain

$$
h=\frac{k}{2}, \quad \bar{h}=0, \quad q=k, \quad \bar{q}=0,
$$

similarly if we start with any other chiral operator in the $\mathcal{N}=2$ vacuum multiplet. To prove that the twist gap with respect to the $\mathcal{N}=2$ Virasoro algebra is zero, it remains to show that this type of spectral flowed operator is not an $\mathcal{N}=2$ descendant of the identity when $c>3$.

We will prove this statement by contradiction. Suppose that every spectral flow image of general chiral generators of the $\mathcal{N}=2$ algebra is an $\mathcal{N}=2$ vacuum descendant. The identity character $\chi_{0}^{\mathrm{NS}}$ must be invariant under spectral flow, which requires

$$
\chi_{0}^{\mathrm{NS}}(\tau, z)=\chi_{0}^{\mathrm{NS}}(\tau, z+\tau) y^{k} q^{\frac{k}{2}} .
$$

For theories with $c>3$, the NS sector vacuum character is given by [17-20]

$$
\begin{aligned}
\chi_{0}^{\mathrm{NS}}(\tau, z)= & q^{-\frac{c-3}{24}} \frac{1-q}{\left(1+y^{\sqrt{\frac{3 k}{c}}} q^{1 / 2}\right)\left(1+y^{-\sqrt{\frac{3 k}{c}}} q^{1 / 2}\right)} \\
& \times \frac{\theta_{3}\left(\tau, \sqrt{\frac{3 k}{c}} z\right)}{\eta(\tau)^{3}} .
\end{aligned}
$$

Recall $\sqrt{3 k / c}$ is the (normalized) $U(1)_{R}$ charge of the supercurrent $G^{+}$which is required to be a positive integer. Then (3.11) simply requires

$$
\begin{aligned}
q^{-\frac{k}{2}} y^{-k}= & \frac{\left(1+y^{\sqrt{\frac{3 k}{c}}} q^{\frac{1}{2}}+\sqrt{\frac{3 k}{c}}\right)\left(1+y^{-\sqrt{\frac{3 k}{c}}} q^{\frac{1}{2}}-\sqrt{\frac{3 k}{c}}\right)}{\left(1+y^{\sqrt{\frac{3 k}{c}}} q^{1 / 2}\right)\left(1+y^{-\sqrt{\frac{3 k}{c}}} q^{\frac{1}{2}}\right)} \\
& \times \frac{\theta_{3}\left(\tau, \sqrt{\frac{3 k}{c}}(z+\tau)\right)}{\theta_{3}\left(\tau, \sqrt{\frac{3 k}{c}} z\right)} .
\end{aligned}
$$

Let us expand both sides around small $q$. Recall that $\theta_{3}(\tau, z)=\sum_{n \in \mathbb{Z}} q^{n^{2} / 2} y^{n}$; thus, $\theta_{3}(\tau, \alpha(z+\tau))$ is dominated by the summand with $n=-\alpha$ and gives (with $\alpha=\sqrt{\frac{3 k}{c}}$ here)

$$
q^{-\frac{k}{2}} y^{-k}=q^{-\frac{3 k}{2 c}} y^{-\frac{3 k}{c}},
$$

which is not possible for $c>3$. 
This implies that if the holomorphic R-symmetry is $U(1)$, all $\mathcal{N}=2$ SCFTs with $c>3$ have vanishing twist gaps with respect to the unextended $\mathcal{N}=2$ algebra. ${ }^{13}$

When $c=3 \hat{c}$ is a multiple of 3 (i.e., $\hat{c} \in \mathbb{Z}$ ) and when $k=c / 3$, the (unextended) $\mathcal{N}=2$ Virasoro algebra is extended by the spectral flow current (3.10) which has $h=\hat{c} / 2$. The spectral flow current and its superpartner, together with the $\mathcal{N}=2$ Virasoro algebra, generate the extended $\mathcal{N}=2$ Virasoro algebra [21]. This is the algebra of, for instance, sigma models with target space Calabi-Yau manifold.

As a corollary, for any $\mathcal{N}=(2,2)$ SCFT whose holomorphic R-symmetry is $U(1)$ (as opposed to $\mathbb{R}$ ), the quantization of the R-charge of the supercurrent (3.8) and that of the level $k \in \mathbb{Z}(2.11)$ imply that the central charge must be rational. In other words, in $\mathcal{N}=(2,2)$ SCFTs with irrational central charges, ${ }^{14}$ the holomorphic R-symmetry must globally be $\mathbb{R}$ (as opposed to $U(1)$ ).

We are now left with the case when the holomorphic $\mathrm{R}$-symmetry is $\mathbb{R}$. The argument in Sec. III A still implies

\footnotetext{
${ }^{13}$ For the $\mathcal{N}=2$ minimal models whose central charge are $c=\frac{3 m}{m+2}$, the vacuum character is different, and is indeed invariant under spectral flow for $k=4 m(m+2)$ or $m(m+2)$ for odd and even $m$, respectively. Correspondingly, the spectral flow currents (3.10) are $\mathcal{N}=2$ descendants of the identity, and the twist gap is nonzero.

${ }^{14}$ In [22], a large class of 2D SCFTs are constructed from compactifications of 4D and 6D SCFTs and their central charges are determined by $c$-extremization. However, all examples considered there with $\mathcal{N}=(2,2)$ supersymmetry turn out to have rational central charges.
}

that the global form of the R-symmetry group is $U(1) \times U(1)$, but each factor of $U(1)$ is not generated by a holomorphic or an antiholomorphic current. As in Sec. III B, we apply integer units of spectral flows for both (nonholomorphic) $U(1)$ 's on the identity to generate a sequence of states with arbitrarily low twists. Since these states have arbitrarily small but nonzero twists, they cannot be $\mathcal{N}=2$ descendants of the identity. Therefore, the twist gap with respect to the (unextended) $\mathcal{N}=2$ Virasoro algebra vanishes even without assuming the holomorphic R-symmetry is globally $U(1)$.

\section{ACKNOWLEDGMENTS}

We thank Igor Klebanov, Petr Kravchuk, Ying-Hsuan Lin, Juan Maldacena, and Xi Yin for interesting discussions. The work of N. B. is supported in part by the Simons Foundation Grant No. 488653. The work of H.O. is supported in part by U.S. Department of Energy Grant No. DE-SC0011632, by the World Premier International Research Center Initiative, MEXT, Japan, by JSPS Grantin-Aid for Scientific Research C-26400240, and by JSPS Grant-in-Aid for Scientific Research on Innovative Areas 15H05895. The work of S.-H.S. is supported by the Simons Collaboration on Ultra-Quantum Matter, which is a grant from the Simons Foundation (Grant No. 651440, N. S.). The work of Y.W. is supported in part by the U.S. NSF under Grant No. PHY-1620059 and by the Simons Foundation Grant No. 488653. We thank the Aspen Center for Theoretical Physics, which is supported by the National Science Foundation Grant No. PHY-1607611, where this work was initiated.
[1] J. L. Cardy, Operator content of two-dimensional conformally invariant theories, Nucl. Phys. B270, 186 (1986).

[2] S. Collier, Y.-H. Lin, and X. Yin, Modular bootstrap revisited, J. High Energy Phys. 09 (2018) 061.

[3] N. Benjamin, H. Ooguri, S.-H. Shao, and Y. Wang, Lightcone modular bootstrap and pure gravity, Phys. Rev. D 100, 066029 (2019).

[4] L. F. Alday and J.-B. Bae, Rademacher expansions and the spectrum of 2d CFT, arXiv:2001.00022.

[5] L. Bhardwaj and Y. Tachikawa, On finite symmetries and their gauging in two dimensions, J. High Energy Phys. 03 (2018) 189.

[6] C.-M. Chang, Y.-H. Lin, S.-H. Shao, Y. Wang, and X. Yin, Topological defect lines and renormalization group flows in two dimensions, J. High Energy Phys. 01 (2019) 026.

[7] Y.-H. Lin and S.-H. Shao, Anomalies and bounds on charged operators, Phys. Rev. D 100, 025013 (2019).
[8] A. Schwimmer and N. Seiberg, Comments on the $N=2$, $N=3, N=4$ superconformal algebras in two-dimensions, Phys. Lett. B 184, 191 (1987).

[9] N. Benjamin, E. Dyer, A. L. Fitzpatrick, and S. Kachru, Universal bounds on charged states in $2 \mathrm{~d}$ CFT and $3 \mathrm{~d}$ gravity, J. High Energy Phys. 08 (2016) 041.

[10] D. Harlow and H. Ooguri, Symmetries in quantum field theory and quantum gravity, arXiv:1810.05338.

[11] J. Polchinski, Monopoles, duality, and string theory, Int. J. Mod. Phys. A 19, 145 (2004).

[12] T. Banks and N. Seiberg, Symmetries and strings in field theory and gravity, Phys. Rev. D 83, 084019 (2011).

[13] A. Maloney and E. Witten, Quantum gravity partition functions in three dimensions, J. High Energy Phys. 02 (2010) 029.

[14] C. A. Keller and A. Maloney, Poincare series, 3D gravity and CFT spectroscopy, J. High Energy Phys. 02 (2015) 080 . 
[15] B. L. Feigin and D. B. Fuchs, Verma modules over the virasoro algebra, Lect. Notes Math. 1060, 230 (1984).

[16] A. Rocha-Caridi, Vertex Operators in Mathematics and Physics, edited by J. Lepowsky, S. Mandelstam, and I. M. Singer (Springer-Verlag, New York, 1985), pp. 451-473, https://doi.org/10.1007/978-1-4613-9550-8_22.

[17] W. Boucher, D. Friedan, and A. Kent, Determinant formulae and unitarity for the $N=2$ superconformal algebras in twodimensions or exact results on string compactification, Phys. Lett. B 172, 316 (1986).

[18] V. K. Dobrev, Characters of the unitarizable highest weight modules over the $N=2$ superconformal algebras, Phys. Lett. B 186, 43 (1987).
[19] E. Kiritsis, Character formulae and the structure of the representations of the $N=1, N=2$ superconformal algebras, Int. J. Mod. Phys. A 03, 1871 (1988).

[20] T. Eguchi and A. Taormina, On the unitary representations of $N=2$ and $N=4$ superconformal algebras, Phys. Lett. B 210, 125 (1988).

[21] S. Odake, Extension of $N=2$ superconformal algebra and Calabi-Yau compactification, Mod. Phys. Lett. A 04, 557 (1989).

[22] F. Benini and N. Bobev, Two-dimensional SCFTs from wrapped branes and c-extremization, J. High Energy Phys. 06 (2013) 005. 